\title{
Determinação da glibenclamida por espectrofotometria derivada no ultravioleta para avaliação do teste ou perfil de dissolução de comprimidos
}

\author{
Janaina Diniz Martins' ${ }^{1}$, Christiane Gino Colu Nery², Gerson Antônio Pianetti', Nilton de Souza \\ Viana-Júnior ${ }^{1}$, Cristina Duarte Vianna-Soares ${ }^{1 *}$
}

'Departamento de Produtos Farmacêuticos, Universidade Federal de Minas Gerais, Fundação Ezequiel Dias/ FUNED, MG

*Correspondência:

C. D. Vianna-Soares

Departamento de Produtos

Farmacêuticos

Faculdade de Farmácia

Universidade Federal de Minas Gerais

Av. Antonio Carlos, 6627/4037

31270-910 - Belo Horizonte - MG,

Brasil

E-mail: cviannas@yahoo.com
A glibenclamida (GLIB) ou gliburida, agente hipoglicemiante oral de segunda geração, da classe das sulfoniluréias, é usada sob a forma de comprimidos para controle do diabetes mellitus. Possui baixa solubilidade aquosa, podendo proporcionar baixa liberação no teste de dissolução de comprimidos e acarretar variabilidades no tratamento. A solução metanólica de GLIB apresenta máximos de absorção em $\lambda 210 \mathrm{~nm}, 227,5 \mathrm{~nm}$ e $300 \mathrm{~nm}$ na região ultravioleta $(U V)$. Após liberaçao de comprimidos, a sua determinação espectrofotométrica na região UV é dificultada devido à baixa concentração proporcionada após o teste de dissolução (comprimidos GLIB $5 \mathrm{mg}$, volume de meio $900 \mathrm{~mL}, 0,56 \mathrm{mg} \%$ ) e à baixa absortividade em comprimento de onda característico, $\lambda$ $300 \mathrm{~nm}$. Por estas razões, até hoje, não existe um método de análise proposto para o teste de dissolução em farmacopéias nacional ou estrangeiras para monografia de glibenclamida comprimidos. A falta de uniformidade nestes procedimentos onera a execução de testes de dissolução para verificação da equivalência farmacêutica de especialidades candidatas a genéricos. Este trabalho propõe a determinação espectrofotométrica de GLIB por derivada de primeira e segunda ordens (máximos $\lambda 238 \mathrm{~nm}$ e $218 \mathrm{~nm}$, respectivamente, em tampão fosfato $0,1 \mathrm{~mol} \mathrm{~L}^{-1}$ ), utilizando comprimidos referência e teste contendo $5 \mathrm{mg}$ de fármaco por dose unitária.

\section{INTRODUÇÃO}

No diabetes mellitus, doença endócrina mais comum, os sintomas são caracterizados por hiperglicemia, metabo- lismo alterado de lipídios, carboidratos e proteínas e por risco aumentado de complicações cardiovasculares. A maioria dos pacientes pode ser clinicamente classificada como portadora de diabetes do tipo I (diabetes mellitus 
insulino-dependente, DMID) ou diabetes mellitus do tipo II (diabetes mellitus não insulino-dependente, DMNID). Segundo Hardman, Limbird (1996), quase todas as formas de diabetes mellitus são geradas por diminuição na concentração circulante de insulina (deficiência de insulina) e por redução na resposta dos tecidos periféricos à insulina (resistência à insulina). Todos os portadores de DMID devem utilizar insulina e para os portadores de DMNID, geralmente, inicia-se uma tentativa de controle das taxas de glicose por meio de dieta alimentar, exercícios ou até a associação destes com o uso de um hipoglicemiante oral.

O hipoglicemiante oral, glibenclamida (Figura 1, GLIB, 1-\{4-[2-(5-cloro-2-metoxibenzamido)etil]benzenossulfonil\}-3ciclo-hexiluréia, MM494,01 $\mathrm{g} \mathrm{mol}^{-1}$ ), também conhecido como gliburida na América do Norte (Merck, 2001; United, 2005), atua estimulando a liberação de insulina endógena, não tendo ação em pacientes que não apresentam função residual de células $\beta$, ligeiramente solúvel em diclorometano e solúvel em dimetilformamida, etanol, metanol e clorofórmio(Clarke, 1986).

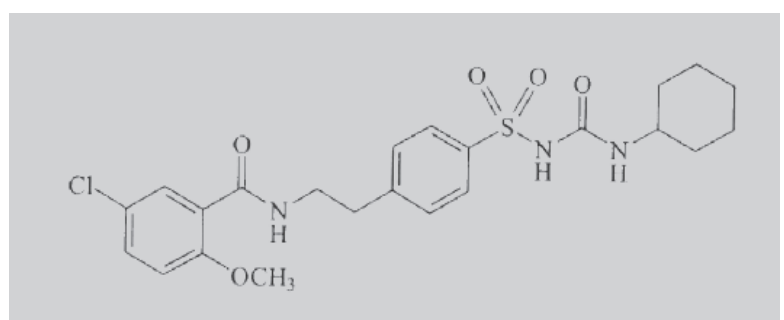

FIGURA 1. Estrutura quimica da glibenclamida, 1-\{4-[2(5-cloro-2-metoxibenzamido)etil] benzenossulfonil\}-3ciclohexiluréia, MM 494,01 $\mathrm{g} \mathrm{mol}^{-1}$.

Técnicas simples de análise quantitativa da glibenclamida, como a volumetria de neutralização em meio aquoso, para a matéria-prima, e outras mais sofisticadas como a cromatografia líquida de alta eficiência, para o produto acabado (comprimidos), estão descritas em monografias de compêndios oficiais como a Farmacopéia Brasileira, $4^{\mathrm{a}}$. edição (Farmacopéia, 2001) e farmacopéias estrangeiras (United States Pharmacopeia, 2005; European Pharmacopeia, 2004; British Pharmacopeia, 2003a,b). No entanto, nenhuma destas farmacopéias propõe um método de análise para o teste de dissolução para glibenclamida comprimidos. Desta forma, onera-se a execução de testes e de perfil de dissolução como forma de comparação com medicamentos referência para verificação da equivalência farmacêutica de especialidades farmacêuticas, candidatas a genéricos (Brasil, 2004). O método espectrofotométrico por derivada (Hackmann et al., 1990; Levillain, Fompeydie, 1986; O’Haver, 1979; Fell, 1978; Talsky et al., 1978) no ultravioleta tem sido largamente empregado nas análises de controle de qualidade de preparações farmacêuticas devido à sua rapidez e simplicidade. Esta técnica é uma alternativa para a determinação de fármacos que sofrem interferência do placebo ou que apresentam valores baixos de absortividade. O presente trabalho avalia a determinação espectrofotométrica de GLIB por derivada de primeira e segunda ordens (máximos $\lambda 238 \mathrm{~nm}$ e $218 \mathrm{~nm}$, respectivamente, em tampão fosfato $0,1 \mathrm{~mol} \mathrm{~L}^{-1}$ ) utilizando comprimidos referência (R) e teste (pilotos A, B, C, D) contendo $5 \mathrm{mg}$ de princípio ativo por dose unitária.

\section{MATERIAL E MÉTODOS}

\section{Material}

O padrão de referência glibenclamida utilizado foi cedido pela Fundação Ezequiel Dias (Funed), apresentando $99,31 \%$ de pureza.

Metanol (Merck), fosfato monobásico de potássio monoidratado e fosfato dibásico de potássio heptaidratado (Vetec) foram utilizados para o preparo de soluções de glibenclamida e de tampão fosfato $0,1 \mathrm{~mol} \mathrm{~L}^{-1}, \mathrm{pH} 7,3$. Comprimidos teste, de mesma formulação, contendo glibenclamida $5 \mathrm{mg}$ (sob diferentes tamanhos de partícula do fármaco, pilotos $\mathrm{A}$ (percentil 90 menor que $120 \mu \mathrm{m}$; percentil 100 menor que $235 \mu \mathrm{m}$ ); $\mathrm{B}$ (percentil 90 menor que $25 \mu \mathrm{m}$; percentil 100 menor que $95 \mu \mathrm{m} \mathrm{e} \mathrm{C}$ (percentil 90 menor que $40 \mu \mathrm{m}$; percentil 100 menor que $165 \mu \mathrm{m}, \mathrm{D}$ (percentil 90 menor que $10 \mu \mathrm{m}$; percentil 100 menor que $85 \mathrm{mðm}$ ) e seu respectivo placebo foram fabricados e gentilmente fornecidos pela Fundação Ezequiel Dias, Belo Horizonte. Comprimidos referência (R) Daonil ${ }^{\circledR}$ (valor declarado $5 \mathrm{mg}$ de glibenclamida por comprimido, Aventis Pharma Ltda.), foram obtidos no comércio local.

Dois espectrofotômetros ultravioleta/visível (Agilent HP8453, simples feixe, definição automática de delta lâmbda e Shimadzu UV160A, duplo feixe, delta lâmbda para primeira ordem $\mathrm{N}=5,4 \mathrm{~nm}$; para segunda ordem $\mathrm{N}=9$, $12,6 \mathrm{~nm}$ ) equipados com modo de derivação interna, cubetas de quartzo de $1 \mathrm{~cm}$ e baixa velocidade de varredura foram utilizados. A faixa de comprimento de onda e a escala de absorvância (assentamento da ordenada) utilizadas estão indicadas em cada figura dos espectros. Todas as medidas foram feitas em triplicata de soluções. O método zero-pico (ZP) foi utilizado para medida das absorvâncias após diferenciação dos espectros.

\section{Métodos}

Preparo da Curva de Ringbom

A curva de Ringbom foi traçada empregando a espectrofotometria em ordem zero no ultravioleta para 
determinação da faixa linear de concentração. As soluções de GLIB utilizadas na faixa de concentração $0,01 \mathrm{mg} \%$ a $3,5 \mathrm{mg} \%$, foram diluídas em tampão fosfato $0,1 \mathrm{~mol} \mathrm{~L}^{-1}$, $\mathrm{pH} 7,3$ para balão volumétrico de $25 \mathrm{~mL}$ a partir de uma solução estoque de GLIB, preparada em metanol ( $25 \mathrm{mg} \%$ ). As medidas foram feitas em comprimentos de onda $225 \mathrm{~nm}$, em triplicata.

\section{Preparo das Curvas de Calibração}

Para o preparo da solução estoque, pesou-se o equivalente a $125 \mathrm{mg}$ de glibenclamida padrão e transferiu-se, quantitativamente, para balão volumétrico de $500 \mathrm{~mL}$, utilizando metanol como solvente. Em seguida, colocou-se em banho de ultra-som até completa solubilização. O volume foi completado com o mesmo solvente, obtendo concentração final correspondente a $25 \mathrm{mg} \%$. Alíquotas apropriadas da solução estoque foram transferidas para balão volumétrico de $25 \mathrm{~mL}$ para obtenção de soluções na faixa de $0,05 \mathrm{mg} \%$ a $1,0 \mathrm{mg} \%$. As soluções diluídas em tampão fosfato $0,1 \mathrm{~mol}$ $\mathrm{L}^{-1}, \mathrm{pH} 7,3$, foram avaliadas no intervalo de comprimento de onda de 200 a $300 \mathrm{~nm}$. A curva de calibração foi traçada a partir de 6 soluções $(n=6)$ preparadas em triplicatas.

\section{Precisão}

O teste de repetibilidade foi realizado através da medida da absorvância em primeira e segunda ordens de derivação, realizado no mesmo dia e com as mesmas condições de análise. A precisão intermediária foi avaliada em primeira e segunda ordens de derivação em equipamentos diferentes. Analisaram-se seis determinações na concentração de trabalho $0,56 \mathrm{mg} \%$ em tampão fosfato $0,1 \mathrm{~mol} \mathrm{~L}^{-1}, \mathrm{pH} 7,3$.

\section{Recuperação}

O teste de recuperação foi realizado utilizando o método de adição de padrões através da medida da absorvância em primeira e segunda ordens de diferenciação nos comprimentos de onda selecionados.

Para o preparo da solução amostra concentrada pesou-se massa de pó dos comprimidos equivalente a 2,5 pesos médios e macerou-se em gral de vidro utilizando-se metanol (aproximadamente $10 \mathrm{~mL}$ ). A suspensão foi transferida para balão volumétrico de $50 \mathrm{~mL}$, utilizando banho de ultra-som para auxiliar a dispersão. O volume foi completado com metanol e a solução foi filtrada, utilizando-se papel de filtro quantitativo. Retiraram-se alíquotas de $0,25 \mathrm{~mL}$, que foram transferidas para balões volumétricos de $25 \mathrm{~mL}$, obtendo concentração final $0,25 \mathrm{mg} \%$.

Alíquotas de 0,25, 0,50 e 1,0 mL de uma solução padrão estoque $(12,5 \mathrm{mg} \% \mathrm{em}$ metanol) foram adicionadas a alíquotas de $0,25 \mathrm{~mL}$ de solução amostra concentrada para balão volumétrico de $25 \mathrm{~mL}$ e o volume completado com tampão fosfato $0,1 \mathrm{~mol} \mathrm{~L}^{-1}, \mathrm{pH} 7,3$. As concentrações teóricas finais em cada balão foram $0,375 \mathrm{mg} \%, 0,50 \mathrm{mg} \%$ e $0,75 \mathrm{mg} \%$. Os resultados obtidos referem-se à média das leituras realizadas em triplicata.

\section{RESULTADOS E DISCUSSÃO}

Para averiguar condições úteis de análise como proposta de procedimento para o teste de dissolução, selecionou-se a solução tampão fosfato $0,1 \mathrm{~mol} \mathrm{~L}^{-1}, \mathrm{pH} 7,3$, como solvente para o preparo das curvas de Ringbom e de calibração, devido à praticidade de também ser utilizada como meio de dissolução para comprimidos. Além disso, o fato de o $\mathrm{pH}$ da solução tampão estar 2 unidades acima do pKa da $\operatorname{GLIB}(5,3)$ contribui para a sua melhor solubilidade, estando o fármaco (ácido fraco) quase totalmente ionizado. Entretanto, testes de solubilidade prévios, mostraram que a GLIB apresentou-se muito pouco solúvel neste diluente (aproximadamente 1:3.300). Por outro lado, soluções de GLIB em metanol formam precipitados, quando em misturas com tampão $0,1 \mathrm{~mol} \mathrm{~L}^{-1}, \mathrm{pH} 7,3$ a partir da concentração 4,5 mg\%. Em contrapartida, soluções de menor concentração de GLIB em metanol são miscíveis em tampão $0,1 \mathrm{~mol} \mathrm{~L}^{-1}, \mathrm{pH} 7,3$.

A solução diluída de $\operatorname{GLIB}(0,56 \mathrm{mg} \%)$, preparada a partir de solução estoque de GLIB em solução de metanol:tampão fostato $0,1 \mathrm{~mol} \mathrm{~L}^{-1}, \mathrm{pH} 7,3(28 \mathrm{mg} \%, 1: 10$ $\mathrm{V} / \mathrm{V})$, apresentou máximos na região ultravioleta em $\lambda 210$ nm, 227,5 nm (ombro) e 300 nm. O máximo em 210 nm, situado na região do ultravioleta baixo, é menos indicado para determinação quantitativa de fármacos em formas farmacêuticas, pois pode sofrer grandes interferências por parte da maioria das substâncias aromáticas. O máximo característico em $\lambda 300 \mathrm{~nm}$ apresentou absortividade muito baixa e, pelo fato de a GLIB ser uma substância de baixa solubilidade, isto torna a análise muito difícil. Portanto, a banda que apresentou absortividade intermediária (região do máximo em $\lambda 225 \mathrm{~nm}$ ) foi selecionada para a realização da curva de Ringbom, medida em transmitância (\%T) e matematicamente transformada em absorvância (100-\%T) como recurso para tornar os dados de transmitância descendentes em uma curva ascendente. $\mathrm{O}$ intervalo sigmoidal de concentração $0,01 \mathrm{mg} \%$ a $3,5 \mathrm{mg} \%$ proporcionou faixa linear de concentração adequada para traçar a curva de calibração na concentração de 0,05 a 1,0 mg\% em tampão fosfato $0,1 \mathrm{~mol} \mathrm{~L}^{-1}, \mathrm{pH} 7,3$.

$\mathrm{Na}$ análise do espectro da substância referência ou padrão GLIB (Figura 2, X) em derivada de primeira ordem, verificaram-se dois mínimos de absorção com boa resolução. O mínimo em $\lambda 238 \mathrm{~nm}$ mostrou-se mais adequado para a determinação da GLIB, apesar de apresentar-se lar- 
go. O espectro de segunda ordem mostrou um máximo e um mínimo bem característicos. O máximo em $\lambda 218 \mathrm{~nm}$ foi selecionado para determinação de GLIB.

Foram construídas três curvas de calibração para cada ordem de derivação em cada espectrofotômetro, em dias diferentes. Para a realização das curvas de calibração, utilizaram-se as absorvâncias das soluções de primeira e segunda ordens de diferenciação, em $\lambda 238 \mathrm{~nm}$ e $218 \mathrm{~nm}$, respectivamente. Os resultados (Tabela I) foram avaliados por meio da regressão linear, método dos mínimos quadrados, equação $\mathrm{Y}=\mathrm{b} \mathrm{X}+\mathrm{a}$, em que $\mathrm{Y}=$ Absorvância/nm (ou Absorvância/nm², e X = Concentração de GLIB (mg\%). Para as duas ordens de derivação, os resultados foram satisfatórios, com coeficiente de correlação superior a 0,99 . A regressão em grupos foi aplicada para decidir se as inclinações das retas diferiam estatisticamente. $\mathrm{O}$ valor da razão de variância encontrada $(2,0)$ foi menor do que o valor F crítico tabelado $(3,89)$. Assim, a hipótese nula foi aceita e construiu-se uma reta comum a partir da média de cada ponto de 3 curvas. Os valores de DPR encontrados, abaixo de $5,0 \%$, permitem que o método validado seja aplicado na quantificação de fármacos para a avaliação de testes de desempenho, como o teste de dissolução.

O método foi aplicado a soluções amostras de comprimidos referência e teste de GLIB $5 \mathrm{mg}$ (R e pilotos A, B, C e D). A seletividade para os lotes pilotos foi confirmada por meio da comparação de superposição espectral entre soluções dos placebos dos lotes piloto com a solução padrão. Em primeira ordem, o mínimo em $\lambda 238 \mathrm{~nm}$, que mostrou ser mais adequado para determinação da GLIB, apesar de largo, encontra-se localizado em posição de maior comprimento de onda e de menor interferência. O máximo, $\lambda 218 \mathrm{~nm}$, em segunda ordem foi selecionado para determinação de GLIB. $\mathrm{Na}$ superposição das soluções padrão $(\mathrm{X})$, pilotos $\mathrm{A}, \mathrm{B}, \mathrm{C}$ e $\mathrm{D}(\mathrm{Y})$ e respectivos placebos $(\mathrm{Z})$, verificou-se a não interferência do placebo na análise, conforme apresentado na Figura 2, em primeira (a) e segunda (b) ordens de derivada.

Os comprimidos referência foram avaliados somente em relação à solução padrão pelo fato de não se dispor do seu placebo. A superposição dos espectros das soluções dos comprimidos referência está apresentada na Figura 3.

Para verificar a repetibilidade e exatidão do método aplicado aos comprimidos piloto de GLIB, realizaram-se os testes de precisão e recuperação (Brasil, 2003) baseandose nas equações de reta obtidas. Para a avaliação da precisão do método utilizaram-se seis determinações na concentração de trabalho $(0,56 \mathrm{mg} \%)$ das quatro formulações piloto (A, B, C e D).

Na Tabela II estão dispostos os valores de teor encontrados das amostras analisadas e seus respectivos valores de desvio padrão relativo (DPR). Em primeira ordem de

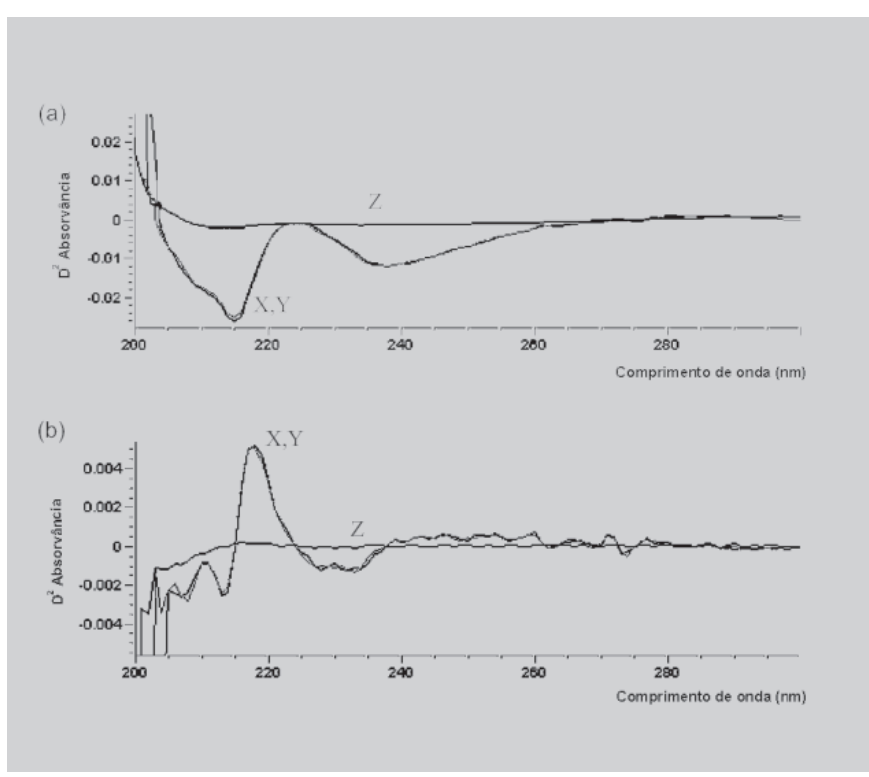

FIGURA 2. Espectro representativo das soluções padrão (X) e comprimidos piloto A, B, C e D (Y) de GLIB na concentração $0,5 \mathrm{mg} \%$, e respectivo placebo $(Z)$ em tampão fosfato $0,1 \mathrm{~mol} \mathrm{~L}^{-1}, \mathrm{pH} 7,3 \mathrm{em}$ primeira (a) e segunda (b) ordens de derivada.

derivação, os resultados obtidos variaram de $97,66 \%$ a $103,03 \%$, DPR de $0,64 \%$ a $0,86 \%$, e de $99,88 \%$ a $111,10 \%$, e DPR de $0,66 \%$ a 1,36\% nos espectrofotômetros HP8453 e UV160A, respectivamente. Em segunda ordem, os resultados obtidos variaram de $97,44 \%$ a $103,50 \%$, com DPR de $0,52 \%$ a $1,22 \%$, e de $92,27 \%$ a $103,05 \%$, e com

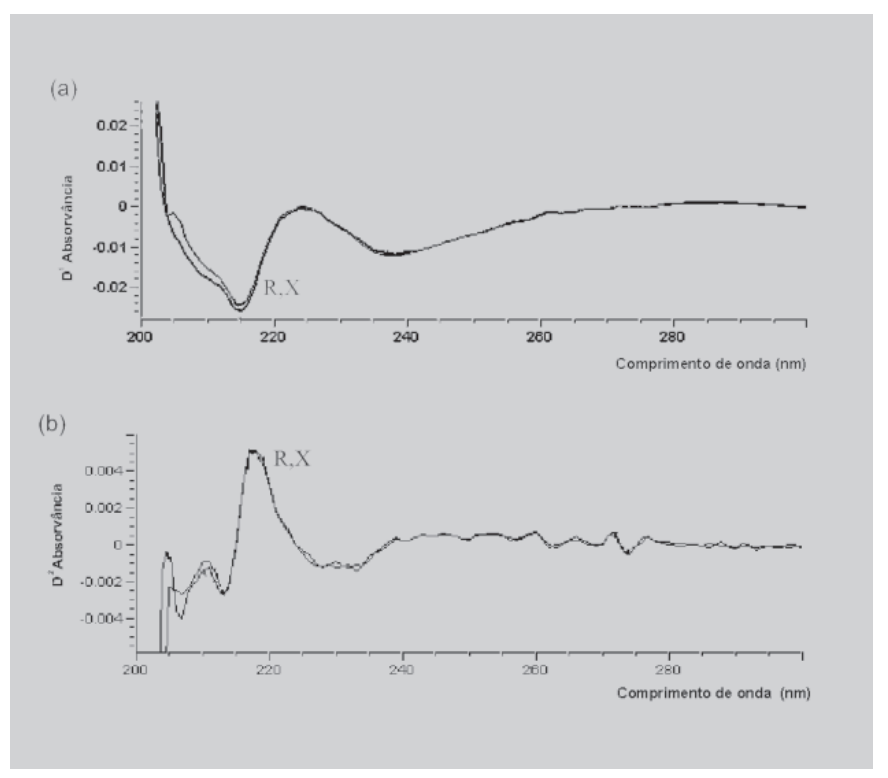

FIGURA 3. Espectros das soluções de GLIB padrão (X) e comprimidos referência $(\mathrm{R})$ na concentração $0,5 \mathrm{mg} \%$ em tampão fosfato $0,1 \mathrm{~mol} \mathrm{~L}^{-1}, \mathrm{pH}$ 7,3 em primeira (a) e segunda (b) ordens de derivada. 
TABELA I - Resultados das curvas de calibração de GLIB em primeira $\left(D_{238}^{1}\right)$ e segunda $\left(D_{218}^{2}\right)$ ordens de diferenciação, em tampão fosfato $0,1 \mathrm{~mol} \mathrm{~L}^{-1}, \mathrm{pH} 7,3$ nos espectrofotômetros HP8453 e UV160A

\begin{tabular}{|c|c|c|}
\hline Parâmetro Espectrofotômetro & Primeira ordem & Segunda ordem \\
\hline HP8453 & $\lambda(238 \mathrm{~nm})$ & $\lambda(218 \mathrm{~nm})$ \\
\hline $\mathrm{b}$ & $-2,10 \times 10^{-2}$ & $9,12 \times 10^{-3}$ \\
\hline $\mathrm{a}$ & $3,22 \times 10^{-4}$ & $-1,32 \times 10^{-4}$ \\
\hline r & 0,9994 & 0,9994 \\
\hline $\operatorname{DPR}(\%)$ & 2,91 & 3,06 \\
\hline$U V 160 A$ & & \\
\hline ent & $9,77 \times 10^{-2}$ & $1,26 \times 10^{-1}$ \\
\hline $\mathrm{a}$ & $-1,45 \times 10^{-4}$ & $-2,64 \times 10^{-3}$ \\
\hline $\mathrm{r}$ & 0,9999 & 0,9995 \\
\hline $\operatorname{DPR}(\%)$ & 1,16 & 2,95 \\
\hline
\end{tabular}

$b$, coeficiente angular; $a$, coeficiente linear; $r$, coeficiente de correlação; DPR, desvio padrão relativo

TABELA II - Resultados médios $(\mathrm{n}=6)$ percentuais de repetibilidade para os comprimidos pilotos de GLIB $5 \mathrm{mg}$ em primeira $\left(D_{238}^{1}\right)$ e segunda $\left(D_{218}^{2}\right)$ ordens de diferenciação, pelo método zero-pico, nos espectrofotômetros HP8453 e UV160A

\begin{tabular}{lllll}
\hline Espectrofotômetro & $\mathrm{A}(\%)$ & $\mathrm{B}(\%)$ & $\mathrm{C}(\%)$ & $\mathrm{D}(\%)$ \\
\hline \multicolumn{1}{c}{ HP8453 } & & & & 101,24 \\
$D_{238}^{1}$ & 98,73 & 97,66 & 103,03 & 0,64 \\
$\mathrm{DPR}^{\mathrm{a}}(\%)$ & 0,86 & 0,80 & 0,82 & 99,27 \\
$D_{218}^{2}$ & 97,44 & 98,14 & 103,50 & 0,72 \\
DPR (\%) & 0,52 & 1,22 & 1,00 & 100,13 \\
\multicolumn{1}{c}{ UV160A } & & & & 1,36 \\
$D_{238}^{1}(\%)$ & 101,27 & 99,88 & 111,10 & 97,71 \\
DPR & 0,92 & 1,15 & 0,66 & 1,12 \\
$D_{218}^{2}$ & 93,52 & 92,27 & 103,05 & 0,57 \\
DPR (\%) & 1,17 & 1,63 & & \\
\hline
\end{tabular}

${ }^{a}$ DPR, desvio padrão relativo

DPR de $0,57 \%$ a $1,63 \%$ nos espectrofotômetros HP8453 e UV160A, respectivamente.

Todos os resultados apresentaram DPR inferior a $2,0 \%$, o que confere uma boa precisão à análise. No entanto, os testes no espectrofotômetro HP8453 mostraram uma faixa de precisão mais estreita do que aquela obtida para os testes de precisão no espectrofotômetros UV160A. A variação de DPR verificada para a primeira ordem foi menor do que para a segunda ordem. Pode-se notar que, em geral, o espectrofotômetro HP8453 mais moderno e sofisticado proporcionou menores valores de DPR, e conseqüentemente maior precisão.

Para avaliação da precisão intermediária, realizaram-se as determinações de GLIB nos diferentes equipamentos. Os valores de DPR para os pilotos A, B, C e D em primeira ordem foram $1,58 \%, 1,40 \%, 3,98 \%, 0,90 \% \mathrm{e}$, para a segunda ordem foram $2,26 \%, 3,47 \%, 0,78 \%, 1,16 \%$ para os pilotos A, B, C e D, respectivamente. Como era de se esperar, estes resultados mostram que a dispersão entre os equipamentos é maior em termos de precisão intermediária. No entanto, são admitidos valores até 4\% para precisão intermediária (Brasil, 2003).

Na Tabela III, apresentam-se os resultados do teste de recuperação das amostras analisadas. Os resultados da recuperação sofreram muita variação nos níveis baixo e alto de concentração. No nível intermediário de concentração a variação foi menor, de $94,57 \%$ a $107,73 \%$, no equipamento HP8453 e no UV160A, de $92,27 \%$ a $111,10 \%$. Estes resultados apontam para variação na exatidão do método entre os equipamentos e conseqüente limitação do método, que pode ser aprimorada. Sugestão para melhorar as porcentagens de recuperação seria a diminuição do vo- 
TABELA III - Resultados ${ }^{\mathrm{a}}$ do teste de recuperação para os comprimidos piloto de GLIB 5 mg, analisados nos espectrofotômetros HP8453 e UV160A

\begin{tabular}{|c|c|c|c|c|c|c|}
\hline Método ZP & $\begin{array}{l}\text { Padrão } \\
\text { adicionado } \\
(\mathrm{mg} \%)\end{array}$ & $\begin{array}{l}\text { Padrão } \\
\text { encontrado } \\
(\mathrm{mg} \%)\end{array}$ & $\begin{array}{l}\text { Recuperação } \\
(\%)\end{array}$ & $\begin{array}{l}\text { Padrão } \\
\text { adicionado } \\
(\mathrm{mg} \%)\end{array}$ & $\begin{array}{l}\text { Padrão } \\
\text { encontrado } \\
(\mathrm{mg} \%)\end{array}$ & $\begin{array}{l}\text { Recuperação } \\
(\%)\end{array}$ \\
\hline & & HP8453 & & & UV160A & \\
\hline & & & Pilot & & & \\
\hline \multirow{3}{*}{$D_{238}^{1}$} & 0,125 & 0,119 & 95,53 & 0,125 & 0,119 & 95,54 \\
\hline & 0,25 & 0,248 & 99,19 & 0,25 & 0,249 & 99,64 \\
\hline & 0,5 & 0,515 & 102,95 & 0,5 & 0,508 & 101,68 \\
\hline \multirow{4}{*}{$D_{218}^{2}$} & 0,125 & 0,118 & 94,57 & 0,25 & 0,113 & 90,75 \\
\hline & 0,25 & 0,254 & 101,49 & 0,5 & 0,240 & 96,02 \\
\hline & 0,5 & 0,528 & 105,62 & 1,0 & 0,507 & 101,30 \\
\hline & & & Pilot & & & \\
\hline \multirow{3}{*}{$D_{238}^{1}$} & 0,125 & 0,121 & 96,46 & 0,125 & 0,133 & 106,46 \\
\hline & 0,25 & 0,247 & 98,70 & 0,25 & 0,246 & 98,27 \\
\hline & 0,5 & 0,513 & 102,53 & 0,5 & 0,502 & 100,32 \\
\hline \multirow[t]{4}{*}{$D_{218}^{2}$} & 0,125 & 0,126 & 100,64 & 0,125 & 0,119 & 94,97 \\
\hline & 0,25 & 0,257 & 102,70 & 0,25 & 0,244 & 97,74 \\
\hline & 0,5 & 0,537 & 107,43 & 0,5 & 0,496 & 99,19 \\
\hline & & & Pilot & & & \\
\hline \multirow{3}{*}{$D_{238}^{1}$} & 0,125 & 0,135 & 107,73 & 0,125 & 0,123 & 98,27 \\
\hline & 0,25 & 0,245 & 98,84 & 0,25 & 0,229 & 95,54 \\
\hline & 0,50 & 0,510 & 101,95 & 0,50 & 0,502 & 100,32 \\
\hline \multirow{4}{*}{$D_{218}^{2}$} & 0,125 & 0,130 & 103,68 & 0,125 & 0,127 & 101,30 \\
\hline & 0,25 & 0,242 & 96,74 & 0,25 & 0,232 & 92,86 \\
\hline & 0,50 & 0,539 & 107,76 & 0,50 & 0,499 & 99,72 \\
\hline & & & Pilot & & & \\
\hline \multirow{3}{*}{$D_{238}^{1}$} & 0,125 & 0,132 & 105,42 & 0,125 & 0,126 & 101,00 \\
\hline & 0,25 & 0,251 & 100,52 & 0,25 & 0,249 & 99,64 \\
\hline & 0,50 & 0,514 & 102,87 & 0,50 & 0,508 & 101,68 \\
\hline \multirow{3}{*}{$D_{218}^{2}$} & 0,125 & 0,130 & 104,10 & 0,125 & 0,124 & 99,19 \\
\hline & 0,25 & 0,256 & 102,50 & 0,25 & 0,240 & 96,02 \\
\hline & 0,50 & 0,536 & 107,14 & 0,50 & 0,493 & 98,66 \\
\hline
\end{tabular}

${ }^{a}$ triplicata

lume de meio de dissolução e, portanto, a elevação do baixo nível de concentração verificado $(0,56 \mathrm{mg} \%, 5 \mathrm{mg}$ em $900 \mathrm{~mL}$ ) para $1 \mathrm{mg} \%$ (5 mg em $500 \mathrm{~mL})$. Não se recomenda, contudo, a mudança das condições de solvente, nem de comprimento de onda, para a sua determinação, pelas razões já apontadas anteriormente. A GLIB, que possui baixa absortividade e solubilidade, apresenta desafios de determinação, principalmente, por espectrofotometria em meio aquoso e quando presente em baixas concentrações. As áreas sob as curvas (AUC) dos gráficos de perfil de dissolução (resultados não mostrados) utilizadas para cálculo de \%ED evidenciam a quantidade de fármaco liberada no meio de dissolução em função do tempo para os comprimidos referência e teste (Tabela IV). Dentre os dois lotes de comprimidos com menor tamanho de partículas, o lote $\mathrm{C}$ mostrou menor eficiência de dissolução em relação ao lote $\mathrm{R}$ em ambas as derivadas. O lote $\mathrm{D}$ mostrou igual eficiência em relação ao lote $\mathrm{R}$ em primeira derivada, porém menor eficiência em relação ao lote $\mathrm{R}$ em segunda derivada.

Nota-se que os comprimidos teste do lote $\mathrm{D}$ apresentam evidências de equivalência farmacêutica com os com- 
TABELA IV - Resultados percentuais ${ }^{\mathrm{a}}$ obtidos por espectrofotometria derivada no ultravioleta (primeira e segunda ordens) para o perfil de dissolução dos comprimidos referência e pilotos $\mathrm{C}$ e $\mathrm{D}$ em tampão fosfato $0,1 \mathrm{~mol} \mathrm{~L}{ }^{-1}, \mathrm{pH} 7,3$, no espectrofotômetro HP8453

\begin{tabular}{lcccccc}
\hline \multirow{2}{*}{$\begin{array}{l}\text { Tempo } \\
(\text { min) }\end{array}$} & \multicolumn{6}{c}{ Média (\%) $\pm \mathrm{dp}^{\mathrm{b}}$} \\
\cline { 2 - 7 } & $D_{238}^{1}$ & $D_{218}^{2}$ & $D_{238}^{1}$ & $D_{218}^{2}$ & $D_{238}^{1}$ & $D_{218}^{2}$ \\
\hline 5 & $41,08 \pm 2,67$ & $48,27 \pm 3,67$ & $27,90 \pm 0,78$ & $26,11 \pm 0,78$ & $31,29 \pm 2,35$ & $29,98 \pm 2,53$ \\
15 & $64,92 \pm 1,43$ & $71,88 \pm 1,17$ & $43,14 \pm 0,83$ & $42,23 \pm 1,02$ & $62,88 \pm 3,89$ & $62,04 \pm 3,57$ \\
30 & $74,19 \pm 1,87$ & $82,97 \pm 1,92$ & $54,72 \pm 1,23$ & $54,91 \pm 1,54$ & $72,84 \pm 1,30$ & $71,76 \pm 0,86$ \\
60 & $79,88 \pm 1,71$ & $87,91 \pm 1,41$ & $66,61 \pm 0,39$ & $65,44 \pm 1,23$ & $81,29 \pm 0,96$ & $80,22 \pm 1,27$ \\
120 & $83,16 \pm 1,61$ & $90,46 \pm 2,10$ & $79,02 \pm 1,24$ & $78,35 \pm 1,08$ & $85,63 \pm 0,89$ & $84,07 \pm 0,70$ \\
150 & $83,68 \pm 1,55$ & $90,90 \pm 1,64$ & $82,64 \pm 1,89$ & $83,64 \pm 1,45$ & $86,28 \pm 1,52$ & $84,54 \pm 1,96$ \\
AUC & 8878,28 & 9797,1 & 7347,75 & 7254,48 & 8886,53 & 8746,95 \\
ED (\%) & 74 & 82 & 61 & 60 & 74 & 73 \\
\hline
\end{tabular}

${ }^{\mathrm{a}}$ sextuplicata; ${ }^{\mathrm{b}} \mathrm{dp}$, desvio padrão

primidos do lote R e é um forte candidato a medicamento genérico.

\section{CONCLUSÃO}

Nas condições descritas, o método apresentou resultados satisfatórios de linearidade e precisão para aplicação em teste desempenho e mostrou diferenças na precisão intermediária e ampla faixa de variações quanto à exatidão, devido à sensibilidade diversa dos equipamentos.

A espectrofotometria por derivada no ultravioleta, tanto para primeira quanto para segunda ordens, mostrouse método fácil e rápido, podendo ser uma alternativa para a determinação de GLIB comprimidos no perfil ou no teste de dissolução.

\section{AGRADECIMENTOS}

Este trabalho foi possível graças ao apoio do CNPq. Os autores agradecem à Fundação Ezequiel Dias/FUNED, pela doação de amostras de matéria-prima e comprimidos de glibenclamida.

\section{ABSTRACT \\ Glibenclamide determination by derivative ultraviolet spectrophotometry for test or dissolution profile assessment in tablets}

Glibenclamide (GLIB) or glyburide, a second-generation oral hypoglycemic drug, is used in the tablet form for the treatment of diabetes mellitus. Because of the low aqueous solubility of this sulfonylurea, a low drug release in the dissolution test may occur, hence, causing variabilility in the treatment. GLIB methanolic solution shows UV absorption maxima in $\lambda 210 \mathrm{~nm}, 227.5 \mathrm{~nm}$ and $300 \mathrm{~nm}$. After released from tablets in the dissolution test, its spectrophotometric determination is difficult due to the low drug concentration in solution (5 mg GLIB tablets, medium volume $900 \mathrm{~mL}, 0.56$ $m g \%)$. In addition, it presents a low absorption in the characteristic wavelength of $\lambda 300 \mathrm{~nm}$. Up to date, there is no method recommendation for GLIB determination in tablet monographs in many of national or foreign official compendia. For this reason, the dissolution test performance lacks uniformity by official recommended procedures, to prove pharmaceutical equivalence to the reference drug, for generic candidates. GLIB spectrophotometric determination is investigated by first and second derivative orders (peaks at $\lambda 238 \mathrm{~nm}$ and $218 \mathrm{~nm}$, respectively in $0.1 \mathrm{~mol} \mathrm{~L}^{-1}$ phosphate buffer solution) using reference and test tablets containing 5 $m g$ of GLIB per unit dose.

UNITERMS: Glibenclamide. Ultraviolet derivative spectrophotometry. Diabetes mellitus. Dissolution profile.

\section{REFERÊNCIAS BIBLIOGRÁFICAS}

BRASIL. Agência Nacional de Vigilância Sanitária. Resolução RE 310 , de $1^{\circ}$ de setembro de 2004, Guia para realização do estudo e elaboração do relatório de equivalência farmacêutica e perfil de dissolução, Diário Oficial da União, Brasília, 3 set. 2004. 5 p.

BRASIL. Agência Nacional de Vigilância Sanitária. Resolução RE 899, 29 de maio de 2003, Guia para validação de métodos analíticos e bioanalíticos. Diário Oficial da União, Brasília, 02 jun. 2003. 16 p. 
BRITISH pharmacopeia, 2003. London: Her Majesty's Stationery Office, 2003a. v.1, p.871-872.

BRITISH pharmacopeia, 2003. London: Her Majesty's Stationery Office, 2003b. v.3, p.2358-2359.

CLARKE, E.G.C., (Ed.). Isolation and identification of drugs. London: Pharmaceutical Press, 1986. p.638-639.

EUROPEAN pharmacopeia. Strasbourg: European Department for Quality of Medicines, 1997. p.898.

FARMACOPÉIA brasileira 4. ed. São Paulo: Atheneu. 1988. pt. 2, n. 3, 2001, p.153, 153-2, 153.1, 153.1-2.

FELL, A.F. Analysis of pharmaceutical dosage forms by second derivative ultraviolet-visible spectrophotometry. Proc. Anal. Div. Chem. Soc., v.15, p.260-267, 1978.

HACKMANN, E.R.M.; BENETON, S.A.; SANTORO, M.I.R.M. Espectrofotometria derivada na análise de fármacos em medicamentos Rev. Port. Farm., v.51, p.712, 1990.
HARDMAN, J.G.; LIMBIRD, L.E., (Eds.). Goodman \& Gilman as bases farmacológicas da terapêutica. 9.ed. Rio de Janeiro: Guanabara Koogan, 1996. p. 1118-1120.

LEVILLAIN, P.; FOMPEYDIE, D. Derivative spectrometry. Principles, advantages and limitations, applications. Analusis, v.14, p.1-20, 1986.

MERCK Index. 13.ed. New Jersey: Merck, 2001. p.798.

O'HAVER, T.C. Potential clinical applications of derivative and wavelength-modulation spectrometry. Clin. Chem., v.25, n.9, p.1548-1553, 1979.

TALSKY, G.; MAYRING, L.; KREUZER, H. Highresolution, higher-order UV/VIS derivative spectrophotometry. Ang. Chem. Int. Ed. English, v.17, n.11, p.785-799, 1978.

UNITED states pharmacopeia 29.ed. Rockville: United States Pharmacopeial Convention, 2005. p.1010-1011.

Recebido para publicação em 11 de maio de 2005. Aceito para publicação em 27 de fevereiro de 2007. 